\title{
BMC Anesthesiology reviewer acknowledgement, 2013
}

Thomas A Rowles

\section{Contributing reviewers}

The editors of BMC Anesthesiology would like to thank all our reviewers who have contributed their time to the journal in Volume 13 (2013).

\begin{tabular}{lll} 
Antonella Agodi & Daniele Biasucci & Davide Cattano \\
Italy & Italy & USA \\
Terrence Allen & Horst Bickel & Vidya Chidambaran \\
USA & Germany & USA \\
Wilson Alves-Do-Prado & Edward Bittner & Daniel Chipman \\
Brazil & USA & USA \\
Barbara Antuna-Puente & Simon Body & Hovig Chitilian \\
Mexico & USA & USA \\
Marco Baciarello & Peter Bragge & Eric Chudler \\
Italy & Australia & USA \\
Angela Bader & Gerhard Brodner & Francois Clergue \\
USA & Germany & Switzerland \\
Sukhminder Bajwa & Donal Buggy & Jean-Marie Conil \\
India & Ireland & France \\
Brian Baldo & John Butterworth & Boris Cruz \\
Australia & USA & Brazil \\
Michael Barrington & Enrico Camporesi & Philipp Dahm \\
Australia & USA & USA \\
Mary Barry & Xavier Capdevila & Abdelazeem Dawlatly \\
Ireland & France & Albania \\
Georgia Beasley & Annalisa Carlucci & Alberto De Armendi \\
USA & Italy & USA \\
Alex Bekker & Michele Carron & Gildasio De Oliveira \\
USA & Italy & USA \\
Dan Benhamou & George Carvalho & Mohamed Bilal Delvi \\
France & Canada & Saudi Arabia \\
\hline & &
\end{tabular}

Correspondence: Tom.Rowles@biomedcentral.com

BioMed Central Floor 6, 236 Gray's Inn Road, London WC1X 8HB, UK 


\begin{tabular}{|c|c|c|}
\hline $\begin{array}{l}\text { Anahat Dhillon } \\
\text { USA }\end{array}$ & $\begin{array}{l}\text { Alejandro González-Ojeda } \\
\text { Mexico }\end{array}$ & $\begin{array}{l}\text { Aaron Kopman } \\
\text { USA }\end{array}$ \\
\hline $\begin{array}{l}\text { Matteo Di Nardo } \\
\text { Italy }\end{array}$ & $\begin{array}{l}\text { Alberto Grassetto } \\
\text { Italy }\end{array}$ & $\begin{array}{l}\text { Keith Kowalczyk } \\
\text { USA }\end{array}$ \\
\hline $\begin{array}{l}\text { Ambra Licia Di Prima } \\
\text { Italy }\end{array}$ & $\begin{array}{l}\text { Robert Greenberg } \\
\text { USA }\end{array}$ & $\begin{array}{l}\text { Morten Tange Kristensen } \\
\text { Denmark }\end{array}$ \\
\hline $\begin{array}{l}\text { Dawn Dillman } \\
\text { USA }\end{array}$ & $\begin{array}{l}\text { Burak Guclu } \\
\text { Turkey }\end{array}$ & $\begin{array}{l}\text { Giovanni Landoni } \\
\text { Italy }\end{array}$ \\
\hline $\begin{array}{l}\text { Prakash Dubey } \\
\text { India }\end{array}$ & $\begin{array}{l}\text { Robert G Hahn } \\
\text { Sweden }\end{array}$ & $\begin{array}{l}\text { Chiara Lazzeri } \\
\text { Italy }\end{array}$ \\
\hline $\begin{array}{l}\text { Leopold Eberhart } \\
\text { Germany }\end{array}$ & $\begin{array}{l}\text { Christiane Hartog } \\
\text { Germany }\end{array}$ & $\begin{array}{l}\text { Francois Lellouche } \\
\text { Canada }\end{array}$ \\
\hline $\begin{array}{l}\text { Roderic Eckenhoff } \\
\text { USA }\end{array}$ & $\begin{array}{l}\text { Avijit Hazra } \\
\text { India }\end{array}$ & $\begin{array}{l}\text { Hendrikus Lemmens } \\
\text { USA }\end{array}$ \\
\hline $\begin{array}{l}\text { Christoph Bernhard Eich } \\
\text { Germany }\end{array}$ & $\begin{array}{l}\text { David Healy } \\
\text { USA }\end{array}$ & $\begin{array}{l}\text { Harald Lenz } \\
\text { Norway }\end{array}$ \\
\hline $\begin{array}{l}\text { Gunnar Elke } \\
\text { Germany }\end{array}$ & $\begin{array}{l}\text { Margareta Hedström } \\
\text { Sweden }\end{array}$ & $\begin{array}{l}\text { Erik Lichnovsky } \\
\text { United Kingdom }\end{array}$ \\
\hline $\begin{array}{l}\text { Martin Esken } \\
\text { Canada }\end{array}$ & $\begin{array}{l}\text { Gregg Homanics } \\
\text { USA }\end{array}$ & $\begin{array}{l}\text { Myong Cheol Lim } \\
\text { South Korea }\end{array}$ \\
\hline $\begin{array}{l}\text { Miguel Ferrer } \\
\text { Spain }\end{array}$ & $\begin{array}{l}\text { Jennifer Hunter } \\
\text { United Kingdom }\end{array}$ & $\begin{array}{l}\text { Steven Lisco } \\
\text { USA }\end{array}$ \\
\hline $\begin{array}{l}\text { Lee Fleisher } \\
\text { USA }\end{array}$ & $\begin{array}{l}\text { Andreas Janata } \\
\text { USA }\end{array}$ & $\begin{array}{l}\text { Carl Lombard } \\
\text { South Africa }\end{array}$ \\
\hline $\begin{array}{l}\text { Carlos Flores } \\
\text { Spain }\end{array}$ & $\begin{array}{l}\text { Vesna Jevtovic-Todorovic } \\
\text { USA }\end{array}$ & $\begin{array}{l}\text { Nadia Lunardi } \\
\text { USA }\end{array}$ \\
\hline $\begin{array}{l}\text { Stuart Forman } \\
\text { USA }\end{array}$ & $\begin{array}{l}\text { Stephanie Jones } \\
\text { USA }\end{array}$ & $\begin{array}{l}\text { Troels Haxholdt Lunn } \\
\text { Denmark }\end{array}$ \\
\hline $\begin{array}{l}\text { Vincent Fraipont } \\
\text { Belgium }\end{array}$ & $\begin{array}{l}\text { Girish Joshi } \\
\text { USA }\end{array}$ & $\begin{array}{l}\text { Carl Lynch } \\
\text { USA }\end{array}$ \\
\hline $\begin{array}{l}\text { Tibor Fulop } \\
\text { USA }\end{array}$ & $\begin{array}{l}\text { David Kaczka } \\
\text { USA }\end{array}$ & $\begin{array}{l}\text { Laxmaiah Manchikanti } \\
\text { USA }\end{array}$ \\
\hline $\begin{array}{l}\text { Javier Garcia-Campayo } \\
\text { Spain }\end{array}$ & $\begin{array}{l}\text { Rebecca Kalman } \\
\text { USA }\end{array}$ & $\begin{array}{l}\text { Maria Martin-Cancho } \\
\text { Spain }\end{array}$ \\
\hline $\begin{array}{l}\text { Allan Garland } \\
\text { Canada }\end{array}$ & $\begin{array}{l}\text { Takashi Kawasaki } \\
\text { Japan }\end{array}$ & $\begin{array}{l}\text { Tadashi Matsuura } \\
\text { Japan }\end{array}$ \\
\hline $\begin{array}{l}\text { Marco Gemma } \\
\text { Italy }\end{array}$ & $\begin{array}{l}\text { Mohammad Reza Khajavi } \\
\text { Iran }\end{array}$ & $\begin{array}{l}\text { Tommaso Mauri } \\
\text { Italy }\end{array}$ \\
\hline $\begin{array}{l}\text { Katja Goetz } \\
\text { Germany }\end{array}$ & $\begin{array}{l}\text { Jeffrey Kirsch } \\
\text { USA }\end{array}$ & $\begin{array}{l}\text { Lynne Maxwell } \\
\text { USA }\end{array}$ \\
\hline $\begin{array}{l}\text { Nermin Gogus } \\
\text { Turkey }\end{array}$ & $\begin{array}{l}\text { Moritz A. Konerding } \\
\text { Germany }\end{array}$ & $\begin{array}{l}\text { Angela Meier } \\
\text { USA }\end{array}$ \\
\hline
\end{tabular}


Pavel Michalek

Czech Republic

Søren Mikkelsen

Denmark

Vincent Minville

France

Constance Monitto

USA

Jane Muret

France

Hiroto Narimatsu

Japan

Lone Nikolajsen

Denmark

Eveline Nueesch

United Kingdom

Yen-Chuan Ou

Taiwan

Mehmet Ozcan

USA

Giovanni Pacini

Italy

Christian Pagnoux

France

Peter Pan

USA

Martyn Parker

United Kingdom

Douglas Paull

USA

Rupert Pearse

United Kingdom

Pernille Petersen

Denmark

Annop Piriyapatsom

USA

Neil Pollock

New Zealand

Valentina Porta

Brazil

Jean-Pierre Quenot

France
Peter Quesenberry

USA

Jacob Raphael

USA

Lars Rasmussen

Denmark

Jochen Renner

Germany

Daniel Reuter

Germany

Sheila Riazi

Canada

Reitze Rodseth

South Africa

Stefano Romagnoli

Italy

Parvin Sajedi

Iran

Michael Sander

Germany

Raffaele Scala

Italy

Dirk Schadler

Germany

Michael Schaefer

Germany

Gereon Schaelte

Germany

Stephan Schug

Australia

Lex Schultheis

USA

Marcus J Schultz

Netherlands

Wolfram Schummer

Germany

Konrad Schwarzkopf

Germany

Karin Sennfält

Sweden

Reza Shariat Moharari

Iran
Martin Soerensen

Denmark

Ju-Tae Sohn

South Korea

Massimiliano Sorbello

Italy

Peter Spieth

Germany

Bernd Stegmayr

Sweden

Bala Subramaniam

USA

Rajeev Subramanyam

USA

Koichi Suehiro

Japan

Richard Szydlo

United Kingdom

Hirotaka Tashiro

Japan

Vladimir Tesar

Czech Republic

Lorenz Theiler

Switzerland

Ronan Thibault

Switzerland

Joseph Tobin

USA

Kamil Toker

Turkey

Mark Tommerdahl

USA

Mei-Yung Tsou

Taiwan

Melek Tulunay

Turkey

Betty Tyler

USA

Mikko Uusi-Oukari

Finland

David Van Duin

USA 
Christian Von Heymann

Germany

Dagmar Westerling

Sweden

Chris Winkelman

USA

Hermann Wrigge

Germany
Victor Xia

USA

Zhongcong Xie

USA

Xu Xuzhong

China

Massimo Zambon

Italy
Sebastian Zaremba

USA

Lin Zou

USA

doi:10.1186/1471-2253-14-7

Cite this article as: Rowles: BMC

Anesthesiology reviewer acknowledgement

2013. BMC Anesthesiology 2014 14:7. 